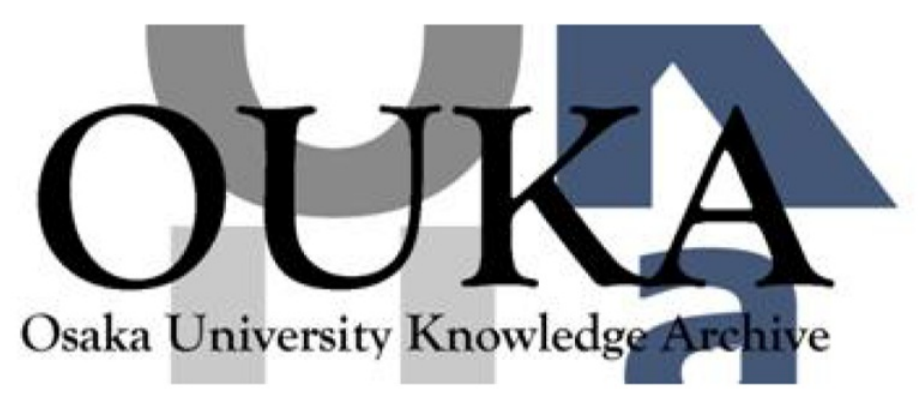

\begin{tabular}{|c|l|}
\hline Title & $\begin{array}{l}\text { Electroabsorption spectroscopy of luminescent } \\
\text { and nonluminescent } \pi \text {-conjugated polymers }\end{array}$ \\
\hline Author(s) & Liess, M. ; Jeglinski, S.; Vardeny, Z. V. et al. \\
\hline Citation & $\begin{array}{l}\text { Physical Review B - Condensed Matter and } \\
\text { Materials Physics. 56(24) p. 15712-p. 15724 }\end{array}$ \\
\hline Issue Date & $1997-12-15$ \\
\hline oaire:version & VoR \\
\hline URL & https://hdl. handle. net/11094/75859 \\
\hline rights & $\begin{array}{l}\text { Copyright (1997) by the American Physical } \\
\text { Society }\end{array}$ \\
\hline Note & \\
\hline
\end{tabular}

Osaka University Knowledge Archive : OUKA

https://ir. Library. osaka-u. ac. jp/

Osaka University 


\title{
Electroabsorption spectroscopy of luminescent and nonluminescent $\pi$-conjugated polymers
}

\author{
M. Liess, ${ }^{*}$ S. Jeglinski, ${ }^{\dagger}$ and Z. V. Vardeny \\ Department of Physics, University of Utah, Salt Lake City, Utah 84112 \\ M. Ozaki and K. Yoshino \\ Department of Electronic Engineering, Faculty of Engineering, Osaka University, 2-1 Yamada-Oka, Suita, Osaka 565, Japan \\ Y. Ding and T. Barton \\ Department of Chemistry, Iowa State University, Ames, Iowa 50011
}

(Received 5 June 1997)

\begin{abstract}
We have measured the quadratic electroabsorption (EA) spectrum of a variety of soluble luminescent and nonluminescent $\pi$-conjugated polymer films in the spectral range of 1.5-4.5 eV. The luminescent polymers include MEH and DOO derivatives of poly(phenylene-vinylene), poly(phenylene ethylene), and polythiophene; the nonluminescent polymers include poly(diethynyl silane) and monosubstituted polyacetylene. All EA spectra show a Stark shift of the low-lying odd-parity exciton $\left(1 B_{u}\right)$ and imply the presence of phonon sidebands. There are also higher-energy bands due to transfer of oscillator strength to even-parity exciton states $\left(A_{g}\right)$, the strongest of which $\left(m A_{g}\right)$ is located at an energy about 1.3 times that of the $1 B_{u}$ exciton in both luminescent and nonluminescent polymers; in the luminescent polymers the EA spectra also show a second prominent $A_{g}$ state $\left(k A_{g}\right)$ at an energy of about 1.6 times that of the $1 B_{u}$. We have successfully fitted the EA spectra by calculating the imaginary part of the third order optical susceptibility, $\operatorname{Im}\left[\chi^{3}(-\omega ; \omega, 0,0)\right]$, using a summation over states model dominated by the ground state, the $1 B_{u}$ exciton, two strongly coupled $A_{g}$ states ( $m A_{g}$ and $k A_{g}$ ), and their most strongly coupled vibrations, using Frank-Condon overlap integrals. A distribution of conjugation lengths, which results in a distribution of excited state energies, was also incorporated into the model. The decomposition of the EA spectra due to the conjugation length distribution was then used to calculate the $1 B_{u}$ exciton polarizability $(\Delta p)$ using first derivative analysis. For the longest conjugation lengths in our films, we found $\Delta p$ to be of order $10^{4}(\AA)^{3}$ in luminescent polymers and $10^{3} \AA^{3}$ in nonluminescent polymers, respectively, in good agreement with recent subnanosecond transient photoconductivity measurements. We also found that the Huang-Rhys parameter of the $1 B_{u}$ exciton varies between 0.25 and 0.9 , being in general smaller for the luminescent polymers. The consequent exciton relaxation energies were calculated to be of order $100 \mathrm{meV}$. [S0163-1829(97)03948-9]
\end{abstract}

\section{INTRODUCTION}

Electroabsorption (EA) spectroscopy, measured using an electric field to modulate the absorption, enhances the "fine structure" in a material's optical absorption spectrum. ${ }^{1}$ An electric-field perturbation applied to the material under investigation creates small changes in the electron wave functions accompanied by small changes in the electronic energy levels, that can consequently be measured as changes in absorption. Early works formulated the theory of electric-field perturbation in semiconductor materials. ${ }^{1-4}$ These theories were developed further and evolved into relatively complete treatments of the Franz-Keldysh band-edge effect and exciton Stark shift. ${ }^{5-24}$ During the same period, electromodulation spectroscopy was developed and applied to semiconductors such as Si and Ge. ${ }^{15-18,25,26}$ The first application of EA spectroscopy to $\pi$-conjugated polymers was reported on samples of crystalline polydiacetylene (PDA), ${ }^{27-29}$ and the strongest spectral feature was explained in terms of the bandedge (Franz-Keldysh) effect. ${ }^{30,31}$ Later work focused on the lower-energy derivativelike features of the EA spectrum; these features were interpreted in terms of the Stark shift of isolated excitons (binding energy $0.5 \mathrm{eV}$ ), pointing to the role of disorder in diminishing the band-edge effects of thin films of PDA derivatives. ${ }^{32-35}$ The first EA spectroscopy in conducting polymer films was applied to the structurally simplest polymer, namely, the trans and cis isomers of polyacetylene $(\mathrm{CH})_{x} ;{ }^{36-39}$ later studies have focused on its oligomers such as $\beta$-carotene. ${ }^{40,41}$ Other conducting polymers have since attracted much attention and numerous EA studies of various conducting polymer films ${ }^{42-47}$ and $\mu$ crystals ${ }^{48,49}$ were made.

Localized excitonic states with large binding energies are characteristic of low-dimensional systems with strong electron correlation effects. ${ }^{50-55}$ In general, theoretical descriptions of conjugated polymers support binding energies on the order of $0.5 \mathrm{eV}$, indicating that electron correlation plays an important role in these systems. ${ }^{56-60}$ The result of these excitonic models is a description of the electronic excited states in conducting polymers in terms of a manifold of localized excitons with large binding energies, the symmetries of which are restricted either to even parity $\left(A_{g}\right)$ or odd parity $\left(B_{u}\right)$. It is interesting to note that in principle the existence of a single, isolated $B_{u}$ exciton $\left(1 B_{u}\right)$ in the excited-state manifold should give rise to an EA blueshift, but this has not been observed experimentally. Using a straightforward application of perturbation theory, the existence of strongly coupled additional $A_{g}$ states, lying above the energy of the 


\begin{tabular}{|l|l|}
\hline \multicolumn{2}{|c|}{ Luminescent $\pi$-conjugated polymers } \\
\hline $\begin{array}{l}\text { 2,5-substituted } \\
\text { poly (p-phenylene vinylene) } \\
\text { - MEH PPV } \\
\text { - DOO PPV }\end{array}$ \\
\hline $\begin{array}{l}\text { Poly (p-phenylene ethynylene) } \\
\text { - PFE }\end{array}$ \\
\hline $\begin{array}{l}\text { Poly(3-alkylthiophene) } \\
\text { - P3DT } \\
- \text { P3OT }\end{array}$
\end{tabular}

\begin{tabular}{|l|l|}
\hline Nonluminescent \\
- $\begin{array}{l}\text { PoES (diethynyl silane) } \\
\text { Poly(thienylenevinylene) } \\
\text { - PTV }\end{array}$ \\
\hline Substituted $(\mathrm{CH})_{x}$
\end{tabular}

FIG. 1. Some of the studied luminescent and nonluminescent $\pi$-conjugated polymers. Their backbone structures are also shown.

$1 B_{u}$ is then necessary to model the observed EA redshift. ${ }^{6}$

In principle, EA spectra can be analyzed by comparison with the first derivative of the linear absorption since the $1 B_{u}$ Stark shift leads to a derivativelike feature. ${ }^{27,28,62}$ Unfortunately, the existing disorder in most polymer films leads to a distribution of polarizabilities and energies of the $1 B_{u}$ exciton, so that a first derivative analysis of the EA spectrum is not possible in many cases. ${ }^{33}$ Still it is possible to model the lowest-energy part of the EA spectra by including derivatives of higher order (Taylor series expansion), but such a phenomenological approach often leads to unrealistic interpretations. ${ }^{37,45}$ In this paper, we will show that a decomposition of the EA spectrum, with first derivatives like Stark shift features for the individual $1 B_{u}$ components, leads to realistic values of the exciton polarizability. This decomposition requires an appropriate modeling of the EA spectra and its conjugation length distribution.

The EA modelling of a summation over states to calculate the third-order nonlinear optical susceptibility $\chi^{(3)}$ (Refs. 63 and 64) is especially useful because it allows us to potentially apply the EA spectroscopy to probe both optically allowed $\left(B_{u}\right)$ and forbidden $\left(A_{g}\right)$ states. Fitting the calculated EA spectrum with the experimental results therefore adds information not only about the $1 B_{u}$ exciton properties such as polarizability, energy, configuration space, and phonon sidebands, but also about the most strongly coupled $A_{g}$ states and the effects of disorder. This highlights an additional weakness of the simple derivative interpretation, since $A_{g}$ states do not contribute to the linear absorption $\alpha(\omega)$, and therefore their contribution to the EA spectrum cannot be

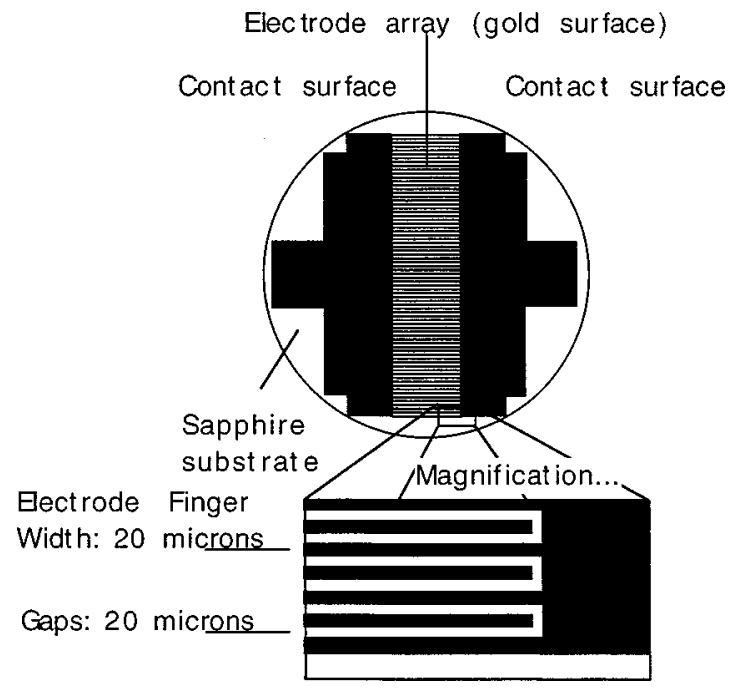

FIG. 2. The electrode configuration used for the EA spectroscopy. The electrodes, of $20 \mu \mathrm{m}$ spacing, were evaporated on the sapphire substrate and photolithographically patterned. The polymer film is spincast on top of the electrodes.

understood from direct derivative analysis of $\alpha(\omega){ }^{34,45,46}$

The polymers studied in this work are either luminescent or nonluminescent soluble conducting polymers films, shown in Fig. 1. Solubility is obtained by using polymers having large side groups, since unsubstituted conjugated polymers are often insoluble. The emissive properties of the luminescent polymers depend on the decay route of the $1 B_{u}$ exciton to the ground state. This is not the case in nonluminescent polymers, where a dipole forbidden state, $2 A_{g}$, lies below the $1 B_{u}$ exciton, supplying a strong alternative nonradiative decay path route. ${ }^{65}$

This paper is organized into five sections. Section II describes the experimental methods, including film casting, electrode configuration on the substrates, and a brief description of the electrical and optical setups. Section III discusses the experimental EA spectra in detail. Section IV contains the EA model and is divided into three subsections: the three most dominant effects included in the EA calculation are discussed separately. In Section IV A the EA spectrum is related to the $\operatorname{Im}\left(\chi^{(3)}\right)$ spectrum with three or four essential states. Section IV B introduces phonon sidebands, and Sec. IV C is devoted to the effect of the conjugation length distribution in the films. In Sec. V A the model calculation is used to fit the EA spectra and to extend the discussion of the most strongly coupled phonons, conjugation length distribution, and the excited-state energies derived from the fits. Section $\mathrm{V}$ B presents the application of the model calculation for decomposition of the absorption and electroabsorption spectra, in order to evaluate the $1 B_{u}$ polarizability and its disorder-induced spectral dependence. The exciton polarizability estimated from the EA spectra is then compared to that inferred from picosecond transient photoconductivity measurements.

\section{EXPERIMENTAL METHOD}

Electrodes for the application of an electric field $(F)$ were deposited on a 0.625 -in.-diameter sapphire disk in an "interlocking finger" geometry, as shown in Fig. 2. A 20- $\mu$ m gap 


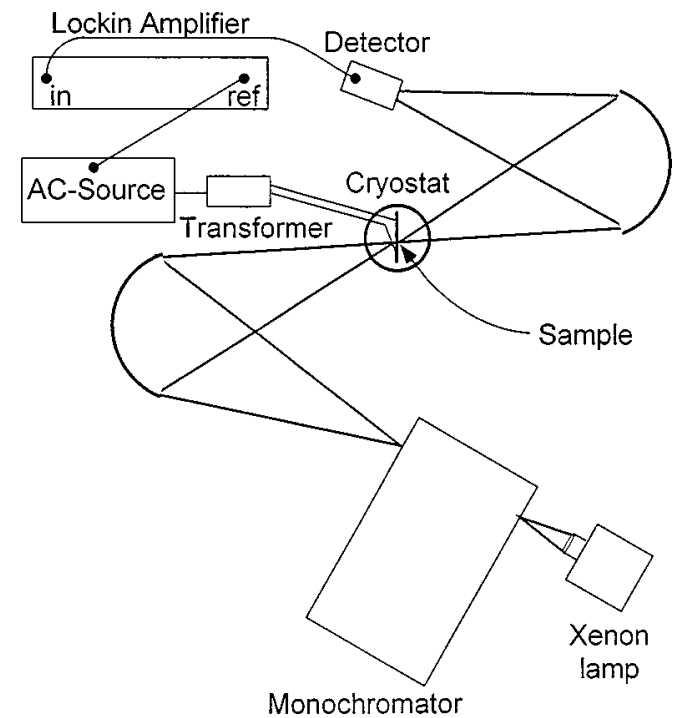

FIG. 3. The optical setup for the EA spectroscopy. Light from a xenon source is wavelength selected by a monochromator and passes through the sample, which is modulated by an AC electric field. The changes in the light transmission are recorded by a lock-in amplifier.

between adjacent electrodes allowed the use of relatively low voltage (hundreds of volts) to achieve $F$ of order $10^{5} \mathrm{~V} / \mathrm{cm}$. The films cast on these electrodes were fabricated from polymer solutions in chloroform or toluene solvents. Care was taken to make the films sufficiently thin (peak optical density $\sim 0.1$ ) to allow measurements at energies of strong absorption.

The experimental setup for the EA measurements is shown in Fig. $3{ }^{61}$ A small sine-wave source was connected to a custom-built step-up transformer (turns ratio of about $1: 130$ ), the output of which was connected to the electrode. The electrodes were contained inside a cryostat for measurements at low temperatures. The electric-field modulation frequencies $f$ were controllable in the range from $250 \mathrm{~Hz}$ to 1 $\mathrm{kHz}$. A mechanically chopped light source (typically a 200-W) tungsten lamp for broadband visible and near infrared, and a $300-\mathrm{W}$ Xe lamp for broad band visible and ultraviolet) was focused on the entrance slit of a computercontrolled $0.25-\mathrm{m} f / 3.5$ monochromator. Long-pass optical filters (to eliminate second-order scattering effects) and neutral density filters (to eliminate excessive incident energy fluence and prevent detector saturation) were used at the monochromator output as needed. The light was refocused on the sample with a mirror, and detected by a UV-enhanced silicon photodiode operated in the photovoltaic mode. This configuration was used to minimize any photodegration and/or heating of the polymer films. The amplified photodiode electrical output was directed to a computer-controlled lock-in amplifier.

For each EA spectrum, the transmission $(T)$ was measured with the mechanical chopper in place and the electric field off. The differential transmission $(\Delta T)$ was subsequently measured without the chopper, with the electric field on, and with the lock-in amplifier set to detect signals at twice the electric-field modulation frequency. The $2 f$ dependency of the EA signal is due to the quadratic nature of EA in materials with definite parity, such as the $\pi$-conjugated
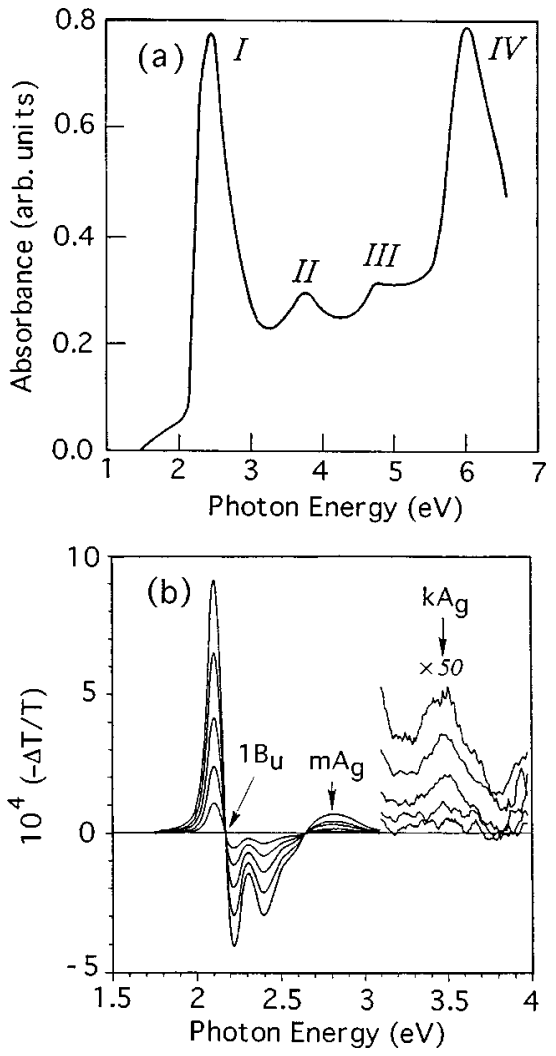

FIG. 4. The optical absorption and EA spectra of a MEH-PPV film at $80 \mathrm{~K}$ at various electric field strengths in the range of 3 $\times 10^{4}-10^{5} \mathrm{~V} / \mathrm{cm}$. Various absorption bands (I-IV) and EA spectral features $\left(1 B_{u}, m A_{g}\right.$, and $\left.k A_{g}\right)$ are assigned.

polymers. ${ }^{27,62} \Delta T$ was then normalized to $\Delta T / T$, which was free of the spectral response function. To a good approximation ${ }^{61}$ the EA signal is related to the imaginary part of the optical third-order susceptibility:

$$
-\Delta T / T=\Delta \alpha d=\frac{4 \pi \omega}{n c} \operatorname{Im}\left[\chi^{(3)}(-\omega ; \omega, 0,0)\right] F^{2} d,
$$

where $F$ is the electric field strength, $d$ is the film thickness, and $n$ is the refractive index. The zero-frequency components in Eq. (1) are from the low-modulation field frequencies which are negligible compared to the frequency $\omega$ of the optical field. We note that, in principle, other effects, such as induced changes in refractive index, thermal and film thickness may contribute to $\Delta T / T$. We found, however, that the sum of all these effects is at most only $5 \%$ of $\Delta T / T .^{61}$

\section{EXPERIMENTAL RESULTS}

The soluble PPV derivative, MEH-PPV, exhibits a typical EA spectrum of luminescent conducting polymers and was chosen as representative of this group of polymers (Fig. 1). The absorption and EA spectra of MEH-PPV are shown in Figs. 4(a) and 4(b), respectively. The absorption spectrum, $\alpha(\omega)$, is composed of four bands: a low-energy band that peaks at $2.4 \mathrm{eV}$ (peak I), two additional small bands with peaks at 3.7 and $4.7 \mathrm{eV}$ (peaks II and III, respectively), and a strong broad band centered at $5.9 \mathrm{eV}$ (peak IV). Very similar absorption spectra have been observed in other PPV derivatives, ${ }^{66-69}$ as well as in derivatives of PT (Ref. 46) and PPE (Fig. 1). ${ }^{70}$ We therefore consider the absorption spec- 
trum in Fig. 4(a) as characteristic of the class of luminescent $\pi$-conjugation polymers, with the side groups affecting $\alpha(\omega)$ only weakly. ${ }^{71}$ Band I is due to the main delocalized $\pi$ - $\pi^{*}$ transition and is most probably the result of an inhomogeneously broadened $1 A_{g}-1 B_{u}$ transition followed by several phonon sidebands. The phonon sidebands are not well resolved in Fig. 4(a) due to the existence of a relatively broad conjugation length distribution in this film. In better, less disordered MEH-PPV films, these vibrational satellites were well resolved in $\alpha(\omega){ }^{72}$ The origin of the 3.7-eV absorption band (II) has been a matter of controversy, assigned either to charge-conjugation symmetry breaking caused by the substitution, or to the existence of short PPV chains in the film. ${ }^{69,73,74}$ No such controversy exists for the remaining bands. Bands III and IV are due to transitions between localized and delocalized states. ${ }^{68}$

Figure 4(b) shows the MEH-PPV EA spectra up to 5.3 $\mathrm{eV}$, at field values $F$ in the range of $10^{4}-10^{5} \mathrm{~V} / \mathrm{cm}$. It was determined $^{61}$ that $\mathrm{EA} \propto F^{2}$, showing the dominance of the quadratic field term in conducting polymers. The EA spectrum is composed of strong features in the range of band I in $\alpha(\omega)$, followed by weak features in the region of bands II and III. Since it is known that localized states have a weak contribution to the EA spectra due to their low polarizability, ${ }^{29}$ the EA result indicates that localized states are indeed involved in bands II and III. This, however, cannot resolve the controversy related to the origin of band II, ${ }^{69,73}$ since band II in the leading models is partially due to localized states.

There are three main EA spectral features in the energy range of band I in $\alpha(\omega)$ : (i) a derivativelike feature with zero crossing at $2.17 \mathrm{eV}$, followed by (ii) three well-resolved vibrational satellites at 2.2,2.4, and $2.6 \mathrm{eV}$, respectively, and (iii) an induced absorption band at $2.8 \mathrm{eV}$. Features (i) and (ii) are the results of a redshifted $1 B_{u}$ exciton energy, and its phonon sidebands (Stark shift). These features are more easily observed in EA than in absorption because of the dependence of the exciton polarizability on the conjugation length $(\mathrm{Sec} . \mathrm{V})$. These samples are disordered and show a preponderance of shorter conjugation lengths. ${ }^{72}$ Since absorption probes all conjugation lengths, the dominance of the shorter chains leads to a mostly featureless absorption spectrum. Electroabsorption, on the other hand, is a $\chi^{(3)}$ process, and therefore preferentially probes the remaining longer conjugation lengths. ${ }^{70}$ This gives rise to the sharper spectral features and also explains the emergence of the phonon side bands. The EA band (iii) at $2.8 \mathrm{eV}$ does not have any corresponding spectral feature in $\alpha(\omega)$, indicating that it is most probably due to an even parity state $\left(m A_{g}\right)$. Such a state would not show up in $\alpha(\omega)$ since the optical transition $1 A_{g} \rightarrow m A_{g}$ is forbidden. We relate band (iii) in EA to transfer of oscillator strength from the allowed $1 A_{g} \rightarrow 1 B_{u}$ transition [band I in $\alpha(\omega)$ ] to the forbidden $1 A_{g} \rightarrow m A_{g}$ transition, caused by the symmetry-breaking external electric field. ${ }^{70} \mathrm{~A}$ similar, smaller band is seen in the EA spectrum at $3.4 \mathrm{eV}$. We attribute this band to a second $A_{g}$ state $\left(k A_{g}\right)$ with weaker polarizability, related to a weaker coupling to the lower $1 B_{u}$ state.

Similar spectral features as in Fig. 4(b) are seen in the EA spectra of other luminescent polymers, such as DOO-PPV, DBO-PPE, and P3DT, shown in Fig. 5. All exhibit a sharp derivativelike feature at low energy, followed by a series of

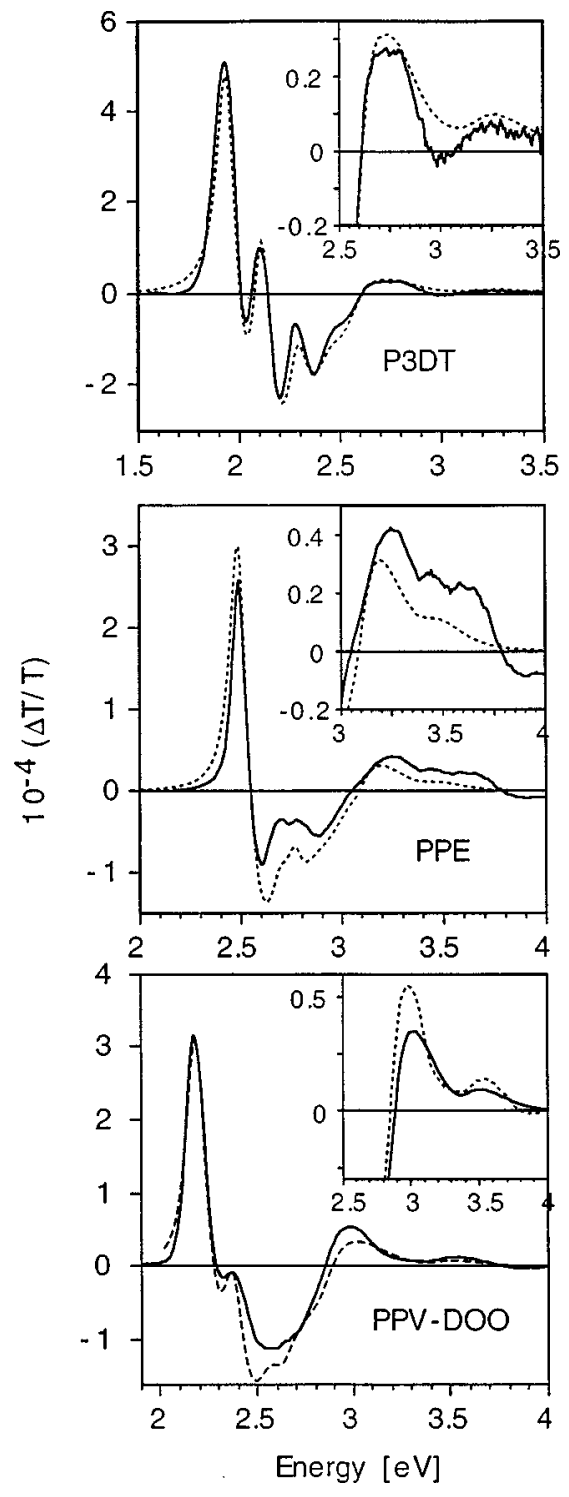

FIG. 5. EA spectra of three luminescent $\pi$-conjugated polymers (P3DT, PPE, and DOO-PPV) measured at $80 \mathrm{~K}$ with $F$ $=10^{5} \mathrm{~V} / \mathrm{cm}$ (full lines) and their theoretical fits (dashed lines). The insets show in more detail the EA features $\left(m A_{g}\right.$ and $\left.k A_{g}\right)$ at high photon energy.

phonon sidebands followed by a relatively strong, positive EA band at higher energies. In all cases a second, weaker band is seen at even higher energies, which we attribute to the $k A_{g}$ state.

As explained above in the Introduction, the most significant difference between the classes of luminescent and nonluminescent conjugated polymers is the presence of an evenparity $\left(2 A_{g}\right)$ state below the $1 B_{u}$ exciton in the latter class of polymers. ${ }^{65}$ Nevertheless, the EA spectra of three nonluminescent polymers, PDA-4BCMU, PDES, and S- $(\mathrm{CH})_{x}$ (shown in Fig. 6) are not qualitatively different from those of the luminescent polymers shown in Fig. 5. We note, however, the existence of an EA low-energy tail below the $1 B_{u}$ feature, which, in principle, may be due to either a broader conjugation length distribution, or the effect of a weakly coupled $2 A_{g}$ below the $1 B_{u}$ state. $^{45,75} \mathrm{We}$ also note the lack of a $k A_{g}$ feature in the EA spectra of this polymer class. 

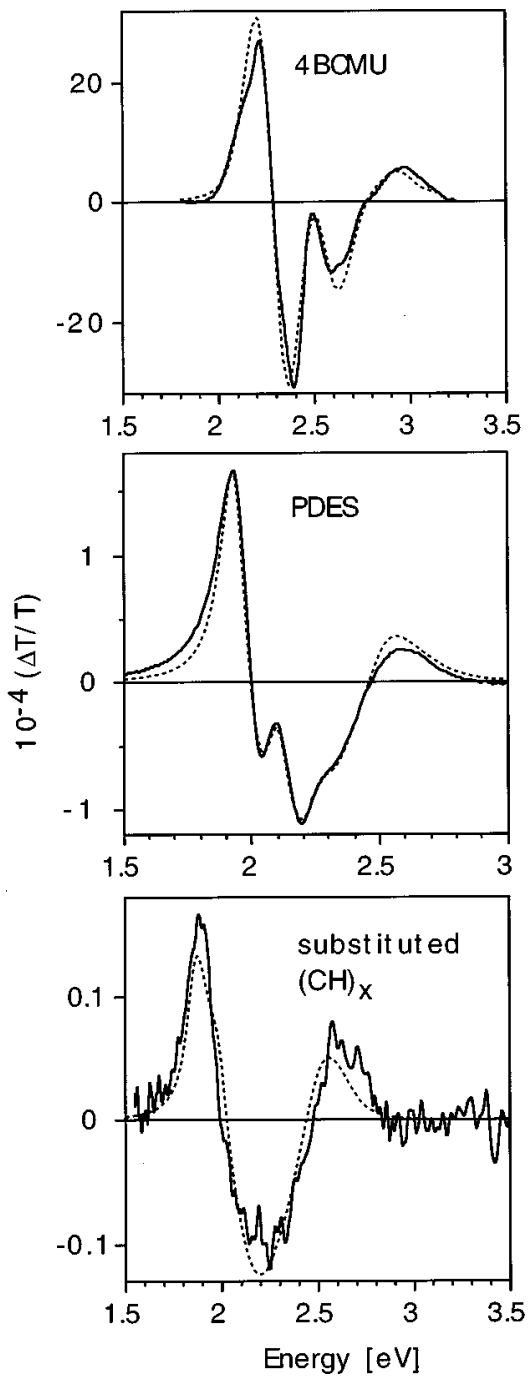

FIG. 6. Same as in Fig. 5 but for three nonluminescent polymers [PDA-4BCMU (Ref. 34), PDES, and substituted $(\mathrm{CH})_{x}$ ].

\section{MODEL CALCULATIONS}

To apply a model for the polymers EA spectra based on $\chi^{(3)}$, we assume that $-\Delta T / T$ is equal to the change in the absorption coefficient $\Delta \alpha d$. It can be shown ${ }^{61}$ that

$$
-\frac{\Delta T}{T}=C_{1} \Delta n+C_{2} \Delta \alpha+\Delta \alpha d,
$$

where $C_{1}$ and $C_{2}$ are optical constants that express a fieldmodulated change in transmission due to a field-induced change in reflectivity of the sample. These terms can be neglected in conducting polymer films, ${ }^{61}$ so that $-\Delta T / T$ $=\Delta \alpha d$ for the films studied here.

\section{A. Essential states in $\chi^{(3)}$ calculation}

The summation over states (SOS) model by Orr and Ward ${ }^{63}$ was used for the calculation of the third-order optical susceptibility $\chi^{(3)}\left(-\omega_{\sigma} ; \omega_{1}, \omega_{2}, \omega_{3}\right)$. This particular model is useful because its formulation is not affected by singularities as are some other common formulations. ${ }^{64}$

Progress in organic nonlinear optics has recently established that a limited three-level model of the ground state, the first allowed $1 B_{u}$ and a particularly strong $A_{g}$ state, the $m A_{g}$, seems to account for $\chi^{(3)}$ spectrum of many molecules. ${ }^{76-80}$ For the one-dimensional centrosymmetrial conducting polymers the SOS model for $\chi^{(3)}$ takes the form $^{80}$

$$
\chi^{(3)} \approx K\left(-\mu_{01}^{4} D_{a}+\mu_{01}^{2} \mu_{12}^{2} D_{b}\right),
$$

where $\mu_{i j}$ is the transition dipole moment between states $i$ and $j ; 0$ is the $1 A_{g}$ state, 1 is the $1 B_{u}$ state, 2 is the $m A_{g}$ state, $\mu_{02}$ is identical to zero, and $D_{a}$ and $D_{b}$ each represents four terms with energy denominators that depend on the photon energy $\hbar \omega$. The factor $K$ is a result of intrinsic permutation symmetry requirements. When $\hbar \omega$ is less than $E\left(1 B_{u}\right)$, the $D$ terms in Eq. (3) are all positive.

Linear molecules and polymers can be grouped according to which term in Eq. (3) dominates the three essential states model. The first term in Eq. (3) gives a negative, nonresonant $\chi^{(3)}$ that has not been found to dominate the optical nonlinearities of polymers. Moreover, as shown in Fig. 7(a), this term results in a blueshift EA Stark effect of the $1 B_{u}$ state, which has not been observed in conducting polymers. The second term in Eq. (3) is positive and results in EA redshift of the $1 B_{u}$ [Fig. 7(b)], in agreement with the data. Since both $D_{a}$ and $D_{b}$ contribute to $\chi^{(3)}$, we conclude from the data that the $D_{b}$ term dominates $\chi^{(3)}$ in conducting polymers. This can be explained only if $\mu_{12} \gg \mu_{01}$ and the energy difference $\left[E\left(m A_{g}\right)-E\left(1 B_{u}\right)\right]<E\left(1 B_{u}\right)$. These are already meaningful conclusions that can be drawn from limited inspection of the data in Figs. 4-6.

With the simplifications of Eq. (3), the eight terms of the complete SOS model ${ }^{63,64}$ can be written as

$$
\begin{aligned}
\chi_{A}^{(3)}\left(-\omega_{\sigma} ; \omega_{1}, \omega_{2}, \omega_{3}\right)= & \frac{N}{\hbar^{3}} \mathbf{P}\left(\omega_{1}, \omega_{2}, \omega_{3}\right)\left[M _ { 1 } \left(D_{1}+D_{2}\right.\right. \\
& \left.+D_{3}+D_{4}\right)-M_{2}\left(D_{5}+D_{6}+D_{7}\right. \\
& \left.\left.+D_{8}\right)\right]
\end{aligned}
$$

where $\hbar \omega_{\sigma}$ is the "test" photon energy, $\omega_{1}$ to $\omega_{3}$ are electric-field frequencies, $M_{1}=\mu_{01}^{2} \mu_{12}^{2}, M_{2}=\mu_{01}^{4}$ [Eq. (3)], and

$$
\begin{gathered}
D_{1}^{-1}=\left(E_{1 B_{u}}-\omega_{\sigma}\right)\left(E_{m A_{g}}-\omega_{1}-\omega_{2}\right)\left(E_{1 B_{u}}-\omega_{1}\right), \\
D_{2}^{-1}=\left(E_{1 B_{u}}^{*}+\omega_{3}\right)\left(E_{m A_{g}}-\omega_{1}-\omega_{2}\right)\left(E_{1 B_{u}}-\omega_{1}\right), \\
D_{3}^{-1}=\left(E_{1 B_{u}}^{*}+\omega_{1}\right)\left(E_{m A_{g}}^{*}+\omega_{1}+\omega_{2}\right)\left(E_{1 B_{u}}-\omega_{3}\right), \\
D_{4}^{-1}=\left(E_{1 B_{u}}^{*}+\omega_{1}\right)\left(E_{m A_{g}}^{*}+\omega_{1}+\omega_{2}\right)\left(E_{1 B_{u}}^{*}+\omega_{\sigma}\right), \\
D_{5}^{-1}=\left(E_{m A_{g}}-\omega_{\sigma}\right)\left(E_{m A_{g}}-\omega_{3}\right)\left(E_{1 B_{u}}-\omega_{1}\right), \\
D_{6}^{-1}=\left(E_{m A_{g}}-\omega_{3}\right)\left(E_{1 B_{u}}^{*}+\omega_{2}\right)\left(E_{1 B_{u}}-\omega_{1}\right), \\
D_{7}^{-1}=\left(E_{m A_{g}}^{*}+\omega_{\sigma}\right)\left(E_{m A_{g}}^{*}+\omega_{3}\right)\left(E_{1 B_{u}}^{*}+\omega_{1}\right), \\
D_{8}^{-1}=\left(E_{m A_{g}}^{*}+\omega_{3}\right)\left(E_{1 B_{u}}-\omega_{2}\right)\left(E_{1 B_{u}}^{*}+\omega_{1}\right),
\end{gathered}
$$




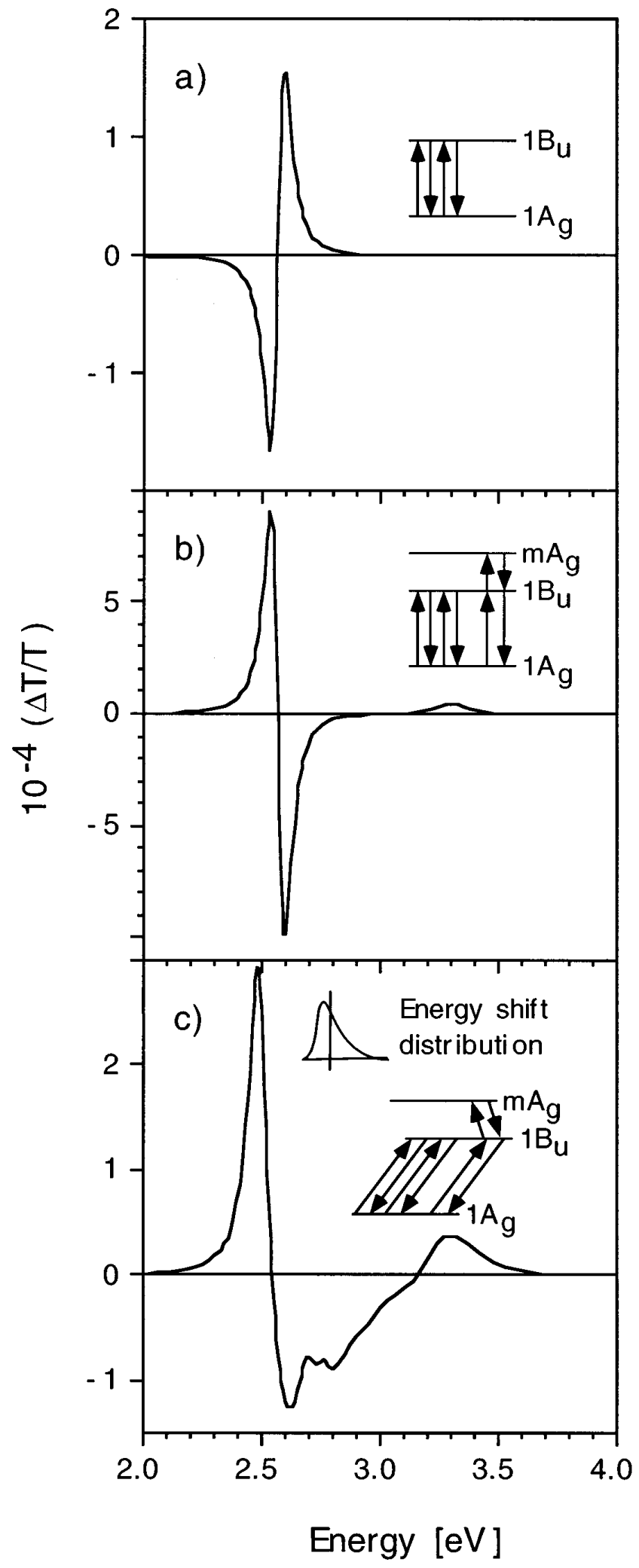

FIG. 7. Various SOS approximations for calculating EA spectra (see text for details). (a) EA spectrum using SOS with two electronic states $\left(1 A_{g}\right.$ and $1 B_{u}$ shown in the inset), where only SOS path (a) in Eq. (3) is allowed. (b) Same as in (a) but for three states $\left(1 A_{g}, 1 B_{u}\right.$, and $m A_{g}$ as shown in the inset), where paths (a) and (b) in Eq. (3) are now allowed. (c) Same as in (b) but the $1 B_{u}$ and $m A_{g}$ states are shifted in configuration coordinate space (lower inset) and a distribution of energy states (upper inset) is considered.

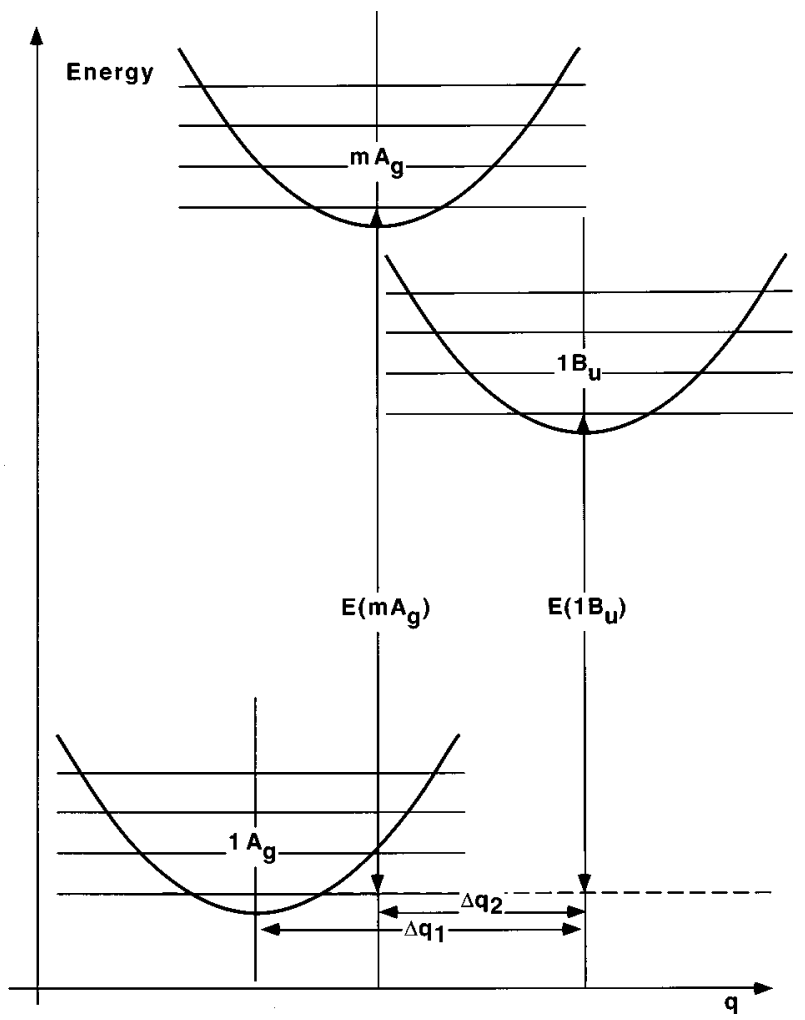

FIG. 8. The three essential states and their coupled vibrations used in the SOS model $\left(1 A_{g}, 1 B_{u}\right.$, and $\left.m A_{g}\right)$ shown in the configurational coordinate $(q)$ space. The shifts $\Delta q_{1}$ for $1 B_{u}$ and $\Delta q_{2}$ for $m A_{g}$ are assigned.

where the terms associated with $M_{1}$ are for pathway (b) and the terms associated with $M_{2}$ stand for pathway (a) in Eq. (3), $\mathbf{P}\left(\omega_{1}, \omega_{2}, \omega_{3}\right)$ in Eq. (4) is the permutation operator, and $E_{1 B_{u}}=\omega_{1 B_{u}}+i \Gamma, E_{m A_{g}}=\omega_{m A_{g}}+i \Gamma$, where $\Gamma$ is the excitedstate energy broadening due to the finite lifetime and inhomogeneous broadening caused by disorder in the film.

Together with the permutation operator $\mathbf{P}\left(\omega_{1}, \omega_{2}, \omega_{3}\right)$, the 8 denominators in Eq. (4) produce 48 different terms. The most resonant denominators, however, which make the strongest contribution to the EA spectrum, are $D_{1}$ and $D_{2}$ for pathway (b) and $D_{5}$ and $D_{6}$ for pathway (a). The terms in Eqs. (5)-(12) were modified to include the effects of vibrational contributions and conjugation length distribution. This is demonstrated below for $D_{1}$ and $D_{5}$ terms only, although all 48 denominators were used in the complete model calculation.

\section{B. Vibrational effects}

To include the effect of the strongly coupled vibrations in the SOS model, each electronic transition is associated with a possible change in the number of coupled phonons. We use the adiabatic approximation in which the transition amplitude between state $A$ with $n_{1}$ phonons and state $B$ with $n_{2}$ phonons is a product of the electronic $\langle A|\mu| B\rangle$ and the "phononic" $\left\langle n_{1}|\Delta q| n_{2}\right\rangle$ transition amplitude. Here $\Delta q$ is the relative displacement of the excited electronic state parabolas (Fig. 8) in the configuration coordinate space, which was introduced to the model [Fig. 7(c)]. The Franck-Condon factor $F_{n_{1}, n_{2}}$ (Ref. 79) is used to calculate the phononic tran- 
sition amplitude from $n_{1}$ to $n_{2}$ phonon states. $F_{n_{1}, n_{2}}$ depends on the overlap integral between the two respective vibrational states:

$$
F_{n_{1}, n_{2}}(\Delta q)=\left\langle n_{1}|\Delta q| n_{2}\right\rangle=\int_{-\infty}^{\infty} \Psi_{n_{1}}^{*}(q) \varphi_{n_{2}}(q-\Delta q) d q
$$

where $\Psi_{n_{1}}$ and $\varphi_{n_{2}}$ are the respective harmonic wave functions expressed in the configuration coordinate $q$. The integral [Eq. (13)] can be solved in a closed form in the harmonic approximation with identical curvatures for the two coupled electronic states, so that the two vibrational eigenstates $\Psi_{n}(q)$ and $\varphi_{n}(q-\Delta q)$ are based on the same phonon frequencies: ${ }^{.9}$

$$
\begin{aligned}
F_{n_{1}, n_{2}}(\Delta q)= & \frac{e^{-(\Delta q)^{2} / 4}}{\sqrt{2^{\left(n_{1}+n_{2}\right)} n_{1} ! n_{2} !}} \sum_{r=0}^{r=\min \left(n_{1} ; n_{2}\right)} \\
& \times \frac{2^{r}(-1)^{\left(n_{1}-r\right)} \Delta q^{\left(n_{1}+n_{2}-2 r\right)} n_{1} ! n_{2} !}{r !\left(n_{1}-r\right) !\left(n_{2}-r\right) !} .
\end{aligned}
$$

The introduction of phonon levels extends the SOS model to include summation over different phonon pathways. The vibrational levels were introduced by modifying the energy levels $E_{i}$ with additional phonon energies $n_{i} h \nu$ where $n_{i}$ is the number of the excited phonons of state " $i$," and $h \nu$ is the phonon quantum energy. In a similar fashion, the transition dipole moment $\mu_{i j}$ was modified to include the phonon transition amplitude $F_{n_{1}, n_{2}}$ [Eq. (13)]. Depending on the position of the states in the configuration space, we have observed that for $\Delta q<1$ summation over the four lowest vibrational states for each electronic state covers $75 \%$ to $95 \%$ of the total transition strength. Summation over additional vibrational states was then omitted in our calculation in order to save computing time. When vibrational effects are included, $\chi_{A}^{(3)}$ of Eq. (4) is modified to $\chi_{B}^{(3)}$ as follows:

$$
\begin{aligned}
\chi_{B}^{(3)}\left(-\omega_{\sigma} ; \omega_{1}, \omega_{2}, \omega_{3}\right)= & \frac{N}{\hbar^{3}} \mathbf{P}\left(\omega_{1}, \omega_{2}, \omega_{3}\right) \\
& \times\left[\sum _ { n _ { 1 } ; n _ { 2 } ; n _ { 3 } = 0 \cdots 3 } M _ { 1 B } \left(D_{1}+D_{2}\right.\right. \\
& \left.+D_{3}+D_{4}\right)-\sum_{n_{4} ; n_{5}=0 \cdots 3} M_{2 B}\left(D_{5}\right. \\
& \left.\left.+D_{6}+D_{7}+D_{8}\right)\right],
\end{aligned}
$$

where

$$
\begin{aligned}
M_{1 B}= & \mu_{12}^{2} \mu_{01}^{2} F_{0, n_{1}}\left(\Delta q_{1}\right) F_{n_{1}, n_{2}}\left(\Delta q_{2}\right) F_{n_{2}, n_{3}}\left(-\Delta q_{2}\right) \\
& \times F_{n_{3} 0}\left(-\Delta q_{1}\right)
\end{aligned}
$$

and

$$
M_{2 B}=\mu_{01}^{4} F_{0, n_{4}}^{2}\left(\Delta q_{1}\right) F_{0, n_{5}}^{2}\left(\Delta q_{1}\right) .
$$

Here $\Delta q_{1}$ and $\Delta q_{2}$ are the shifts in the configurational space (Fig. 8) of $1 B_{u}$ and $m A_{g}$, respectively, relative to $1 A_{g}$.

In Eq. (15), all $D$ terms are modified to include the respective vibrational states; for example, $D_{1 B}$ and $D_{5 B}$ are now given by

$$
\begin{aligned}
D_{1 B}^{-1}= & \left(E_{1 B_{u}}+n_{1} \nu-\omega_{\sigma}\right)\left(E_{m A_{g}}+n_{2} \nu-\omega_{1}-\omega_{2}\right) \\
& \times\left(E_{1 B_{u}}+n_{3} \nu-\omega_{1}\right) \\
D_{5 B}^{-1}= & \left(E_{m A_{g}}+n_{4} \nu-\omega_{\sigma}\right)\left(E_{m A_{g}}+n_{4} \nu-\omega_{3}\right) \\
& \times\left(E_{1 B_{u}}+n_{5} \nu+\omega_{1}\right) .
\end{aligned}
$$

\section{Conjugation length distribution}

Mechanical distortion of the polymer chain limits the conjugation length, which figures in the related electronic wave functions, to be a certain fraction of the chain. It is then common practice to assume that the excited-state energies depend on this conjugation length. ${ }^{81}$ The excited electronic energies do not appear as discrete steps in the polymer spectrum, since the disordered environment broadens the energies of any individual conjugation length. ${ }^{82}$ Therefore, a smooth distribution function for the shifted excited energy levels $\Delta E$ is usually assumed. It has been observed that $E$ depends on the conjugation length according to the relation $^{81,83} E_{N}=E_{\infty}+\Delta E_{N}$ where $\Delta E_{N}=C N^{-1}, E_{\infty}$ is the excited-state energy of the infinite chain, $N$ is the number of repeat units within the conjugation length, and $C$ is an empirical parameter. To include the effect of conjugation length on the energy of each excited state, a weighted integral over the shifted energy states with energies $E^{\prime}$ around the mean excited state energy, $E_{n}$, was performed for $\chi^{(3)} \cdot \chi_{B}^{(3)}$ is then modified to $\chi_{C}^{(3)}$ as follows:

$$
\begin{aligned}
\chi_{C}^{(3)}\left(-\omega_{\sigma} ; \omega_{1}, \omega_{2}, \omega_{3}\right)= & \int_{-\delta}^{\delta} W\left(E^{\prime}\right) \chi_{B}^{(3)}\left(E_{1 B_{u}}+E^{\prime} ; E_{m A_{g}}\right. \\
& \left.+E^{\prime} ;-\omega_{\sigma} ; \omega_{1}, \omega_{2}, \omega_{3}\right) d E^{\prime}
\end{aligned}
$$

where $2 \delta$ is the $E^{\prime}$ distribution width around $E_{n}$ and $W\left(E^{\prime}\right)$ is a weight function. The integral over the energy $E^{\prime}$ is in fact equivalent to an integral over a distribution of conjugation lengths with an inhomogeneous energy broadening taking into account that $E_{n}+\delta \geq E_{\infty}+\Gamma$. A description in terms of the latter model can be found in Ref. 61. Since the relative contributions of the energy states in $\chi_{C}^{(3)}$ depends on $E^{\prime}$, the numerical computation of Eq. (20) cannot be simplified by modifying the field frequencies $\omega$ rather than the real energy levels such as $E_{1 B_{u}}+E^{\prime}$ and $E_{m A_{g}}+E^{\prime}$. The function $W\left(E^{\prime}\right)$ in Eq. (20) not only depends on the conjugation length distribution, but is also influenced by the dependence of the electronic polarizability in such a distribution. Conjugationlength distributions that resemble log-normal functions have been shown to be consistent with resonant Raman scattering, ${ }^{81-83}$ and can be argued to be correct on physical grounds. Moreover, we note that third-harmonic-generation measurements have shown $\chi^{(3)}$ to exhibit a superlinear power-law dependence on the conjugation length $N .^{84-87} \mathrm{Al}-$ 


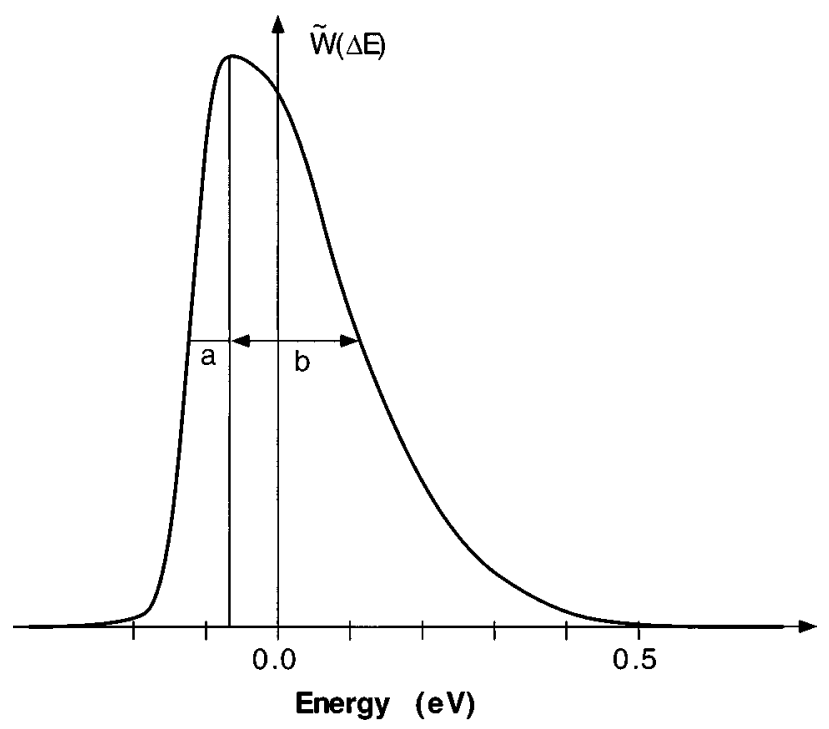

FIG. 9. The asymmetric Gaussian weight function $\widetilde{W}\left(E^{\prime}\right)$ used in the SOS model [Eq. (21)], where the conjugation length distribution is accounted for. The average $E^{\prime}$ is zero, and the $a$ and $b$ parameters causing the asymmetry in $\widetilde{W}$ are assigned.

though EA is equivalent to the dc Kerr effect and not to the third-harmonic generation, it is nevertheless a $\chi^{(3)}$ process [Eq. (1)], and so the assumption of a power-law dependence of $\chi^{(3)}$ on $N$ is justified. The combination of chain length distribution effects on the excited-state energy and $\chi^{(3)}$ conspires $W\left(E^{\prime}\right)$ to saturate at both ends of the $E^{\prime}$ interval. This can be effectively described by an asymmetric $W\left(E^{\prime}\right)$ function, where the functional dependence of the conjugation length distribution and the $\chi^{(3)}$ dependence on $N$ is mapped into the energy space $E^{\prime}$ (Fig. 9). The function $W\left(E^{\prime}\right)$ is then determined by two free parameters, namely, the full width at half maximum (FWHM) $\gamma[\gamma=(a+b)]$ and the asymmetry $\eta[\eta=(b / a)]$, where $a$ and $b$ are defined in Fig. 9. The parameters $\gamma$ and $\eta$ can be adjusted to provide a good fit between the SOS model and the experimental EA spectra.

For the polymers studied here, $W\left(E^{\prime}\right)$ is conveniently approximated by an asymmetric Gaussian function $\widetilde{W}$ as described in Fig. 9, where

$$
\widetilde{W}\left(B, u, E^{\prime}\right)=\frac{1.13}{B} \frac{\exp \left\{-\left[E^{\prime} / B-\xi(u)\right]^{2}\right\}}{1+\exp \left\{-u\left[E^{\prime} / B-\xi(u)\right]\right\}} .
$$

In Eq. (21) $\xi(u)=0.95 /[1+\exp (u)]-0.475$, so that $\int_{\infty}^{0} \widetilde{W}\left(B, u, E^{\prime}\right) d E^{\prime}=\int_{0}^{\infty} \widetilde{W}\left(B, u, E^{\prime}\right) d E^{\prime}=1 / 2$. Hence, the parameters $B$ and $u$ in $\widetilde{W}$ can be varied without changing either the position of the mean energy or the integral contribution of all states to $\chi^{(3)}$. The FWHM $\gamma$ and asymmetry $\eta$ are then numerically evaluated from $\widetilde{W}\left(E^{\prime}\right)$.

\section{MODEL APPLICATIONS}

The SOS model calculation used to simulate the various EA spectra in both luminescent and nonluminescent polymers $^{88}$ includes three (or four) essential states, namely, the states $1 A_{g}, 1 B_{u}$, and $m A_{g}$ (also $k A_{g}$ has been used for luminescent polymers only), four phonon replicas and a chain length distribution function, as described in Eqs. (1), (15), (20), and (21) above. The three essential states of our SOS model are shown in the configuration coordinate space in Fig. 8. There are all together eight free fitting parameters in our SOS model as described in Tables I and II. These are the exciton energies $E\left(1 B_{u}\right)$ and $E\left(m A_{g}\right)$ and their relative displacements $\Delta q$ in the configuration coordinate $q$, namely, $\Delta q\left(1 B_{u}\right)$ and $\Delta q\left(m A_{g}\right)$. One dominant phonon mode $\nu$ in the excited states, the two parameters $\gamma$ and $\eta$ describing the chain length distribution function $\widetilde{W}\left(E^{\prime}\right)$, and the ratio of the dipole moment transitions $\mu_{12} / \mu_{10}$. We fixed the broadening $\Gamma$ in the excited-state energy to be $30 \mathrm{meV},{ }^{41}$ and $\mu_{12} / \mu_{10}=2 .{ }^{41,89}$ The initial values of most fitting parameters were estimated from other measurements. For example, $E\left(1 B_{u}\right)$ was evaluated from $\alpha(\omega)$ spectra, $E\left(m A_{g}\right)$ (and $k A_{g}$ ) was taken from two-photon absorption (TPA) spectra measured in our laboratory, ${ }^{90}$ and $\nu$ was established from published resonant Raman scattering spectra. Excellent fits between the theoretical and experimental EA spectra have been achieved for both luminescent and nonluminescent polymers as seen in Figs. 5 and 6, respectively. The SOS model best-fitting parameters are summarized in Tables I and II for luminescent and nonluminescent polymers, respectively.

Important information can be inferred from the EA spec-

TABLE I. The best fitting parameters for the EA spectra of several luminescent $\pi$-conjugated polymers shown in Figs. 4 and 5 (see text for details).

\begin{tabular}{lcccc}
\hline \hline & PPV-MEH & PPV-DOO & PPE & P3DT \\
\hline$E\left(1 B_{u}\right)(\mathrm{eV})$ & 2.20 & 2.30 & 2.56 & 2.01 \\
$\Delta q_{1}=q\left(1 B_{u}\right)-q\left(1 A_{g}\right)$ & 0.77 & 0.90 & 0.70 & 1.25 \\
$E\left(m A_{g}\right)(\mathrm{eV})$ & 2.80 & 3.00 & 3.18 & 2.67 \\
$m A_{g}$ relative strength $(\%)$ & 60 & 40 & 68 & 30 \\
$E\left(k A_{g}\right)(\mathrm{eV})$ & 3.55 & 3.55 & 3.50 & 3.27 \\
$k A_{g}$ relative strength $(\%)$ & 40 & 60 & 32 & 70 \\
$\Delta q_{2}=q\left(m A_{g}\right)-q\left(1 B_{u}\right)$ & -0.4 & -0.9 & -0.8 & -0.7 \\
$h \nu$ phonon $($ meV $)$ & 190 & 190 & $200^{\mathrm{a}}$ & 173 \\
Chain distribution width $\gamma(\mathrm{eV})$ & 0.11 & 0.20 & 0.14 & 0.10 \\
Distribution asymmetry $\eta$ & 4.5 & 12 & 12 & 20 \\
\hline \hline
\end{tabular}

${ }^{\text {a Due to the triple bond in PPE we fitted the EA with two phonons; the additional phonon energy was }}$ determined to be $275 \mathrm{meV}$. 
TABLE II. The best fitting parameters for the $E A$ spectra of several nonluminescent $\pi$-conjugated polymers shown in Fig. 6 .

\begin{tabular}{lcccc}
\hline \hline & 4BCMU $^{\mathrm{a}}$ & $\mathrm{PTV}^{\mathrm{b}}$ & $s-(\mathrm{CH})_{x}$ & $\mathrm{PDES}$ \\
\hline$\Delta q_{1}=q\left(1 B_{u}\right)-q\left(1 A_{q}\right)$ & 2.29 & 1.83 & 1.98 & 2.01 \\
$\Delta q\left(1 B_{u}\right)$ & 0.8 & 1 & 1.3 & 1 \\
$E\left(m A_{g}\right)(\mathrm{eV})$ & 2.9 & 2.4 & 2.52 & 2.54 \\
$\Delta q_{2}=q\left(m A_{g}\right)-q\left(1 B_{u}\right)$ & 0.4 & 0.8 & 0.0 & 0.2 \\
$h \nu(\mathrm{meV})$ & 270 & 181 & 120 & 167 \\
Distribution & 0.17 & 0.09 & 0.18 & 0.10 \\
$\quad$ width $\gamma(\mathrm{eV})$ & & & & \\
Distribution & 1 & 1.8 & 1 & 12 \\
$\quad$ asymmetry $\eta$ & & & & \\
\hline \hline
\end{tabular}

${ }^{\mathrm{a}}$ From Ref. 34.

${ }^{\mathrm{b}}$ From Ref. 42.

tra and their fits. First, a continuum band at an energy $E_{c}$ above $E\left(1 B_{u}\right)$ is not observed in the EA spectra. This differs from the EA spectra in polydiacetylene (PDA) single crystals, where sharp oscillations at approximately $0.5 \mathrm{eV}$ above $E\left(1 B_{u}\right)$ were identified with the Franz-Keldysh $(\mathrm{FK})$ electric field effect at the continuum band edge. ${ }^{29,30,32}$ The reason for the lack of EA oscillation at $E_{c}$ here may be the existence of disorder and inhomogeneity, which tend to shorten the freecarrier coherence length. ${ }^{32,35}$ This, in turn, eliminates a sharp band edge energy at $E_{c}$, which results in the suppression of the FK oscillation. We note that in the case of PDA, the FK oscillation feature, which dominates the EA spectrum of single crystals, gradually changes into a positive EA band at $m A_{g}$ when the disorder in the film increases. ${ }^{91}$ When generalizing these results to include other conducting polymers we suggest that $E\left(m A_{g}\right)$ may mark a lower limit for the continuum band threshold. With this in mind, it is interesting to note that in all samples (Tables III and IV) we found $E\left(m A_{g}\right) / E\left(1 B_{u}\right) \simeq 1.3 \pm 0.06$, regardless of whether the polymer is luminescent or not. This is significant since theory predicts that $m A_{g}$ is much closer to $1 B_{u}$ in nonluminescent polymers with small effective dimerization. ${ }^{30,31}$ This may indicate that what is dubbed here $m A_{g}$, with a positive band in the EA spectra, may in fact be related to a continuum band threshold. Assuming that a positive band in EA replaces the FK oscillation due to disorder in these films, ${ }^{91}$ a lower limit for the exciton binding energy, $E_{b}$, may be directly found from the EA analysis, where

TABLE III. Additional characterization of the electronic excited states obtained from the fits to the EA spectra of luminescent $\pi$ conjugated polymers given in Table I. $S$ is the $1 B_{u}$ Huang-Rhys parameter, $E_{r}$ is the $1 B_{u}$ relaxation energy, $E_{b}\left[=E\left(m A_{g}\right)\right.$ $\left.-E\left(1 B_{u}\right)\right]$ is the exciton binding energy, and $\delta E\left[=E\left(k A_{g}\right)\right.$ $\left.-E\left(m A_{g}\right)\right]$ is the continuum bandwidth.

\begin{tabular}{lcccc}
\hline \hline & PPV-MEH & PPV-DOO & PPE & P3DT \\
\hline$E\left(m A_{g}\right) / E\left(1 B_{u}\right)$ & 1.27 & 1.30 & 1.48 & 1.33 \\
$S$ & 0.17 & 0.4 & 0.25 & 0.78 \\
$E_{r}(\mathrm{meV})$ & 70 & 65 & 50 & 140 \\
$E_{b}(\mathrm{eV})$ & 0.6 & 0.7 & 0.6 & 0.6 \\
$\delta E(\mathrm{eV})$ & 0.7 & 0.6 & 0.4 & 0.6 \\
\hline \hline
\end{tabular}

TABLE IV. Same as in Table III but for nonluminescent polymers given in Table II.

\begin{tabular}{lcccc}
\hline \hline & 4BCMU & PTV & $s-(\mathrm{CH})_{x}$ & PDES \\
\hline$E\left(m A_{g}\right) / E\left(1 B_{u}\right)$ & 1.27 & 1.31 & 1.33 & 1.26 \\
$S$ & 0.32 & 0.5 & 0.85 & 0.5 \\
$E_{r}(\mathrm{meV})$ & 86 & 90 & 100 & 83 \\
$E_{b}(\mathrm{eV})$ & 0.6 & 0.6 & 0.5 & 0.5 \\
\hline \hline
\end{tabular}

$$
E_{b} \simeq E\left(m A_{g}\right)-E\left(1 B_{u}\right) .
$$

$E_{b}$ defined this way is also given in Tables III and IV for various polymers. $E_{b}$ is seen to be between 0.5 and $0.7 \mathrm{eV}$ for most polymers, ${ }^{92}$ showing the important role of the longrange electron-electron interaction, regardless of whether the polymer is luminescent or not.

In all EA spectra of luminescent conducting polymers we see a second even-parity state, namely, the $k A_{g}$, which is apparent at energies $E\left(k A_{g}\right)>E\left(m A_{g}\right)$. We also note that a second, relativity strong $A_{g}$ state appears above the $m A_{g}$ in the TPA spectra of luminescent conducting polymers. ${ }^{90}$ In fact, the $A_{g}$ states in the TPA spectra are in excellent agreement with those measured by EA in this work, strengthening the interpretation of the positive EA bands seen above $1 B_{u}$ as being due to $A_{g}$ states. We define $\delta E$ as

$$
\delta E=E\left(k A_{g}\right)-E\left(m A_{g}\right)
$$

and calculate from the EA spectra $\delta E$ for luminescent polymers as shown in Table III. For most polymers we found $\delta E \simeq 0.6 \mathrm{eV}$. At this point we may speculate as to the origin of $k A_{g}$. Some theoretical models predict the existence of a second $A_{g}$ state with energy above $E\left(m A_{g}\right)$, which is strongly coupled to the $1 B_{u}$ exciton. ${ }^{41}$ Others ${ }^{43,71}$ identify it as the biexciton state, $B X$. However, it is not clear whether such a state would directly contribute to the EA spectrum since it is composed of two excitons, which therefore cannot be generated by a single photon even if parity is not conserved due to a strong external electric field, as in EA. Another possibility for the origin of the $k A_{g}$ is the shape of the continuum band density-of-state function, $D(E)$. It is well known that $D(E)$ in one dimension (1D) has two Van Hove singularities peaked at the two continuum band edges at $E_{c}$ and $E_{c}+\delta E$, respectively, where $\delta E$ is the width of the continuum band. ${ }^{93}$ As we have discussed above, a continuum band is not a proper description of the electronic states in our conducting polymer films due to disorder, finite-size effects, and inhomogeneity. However, a group of $A_{g}$ states may still mark the two suppressed 1D singularities of the continuum band, where $m A_{g}$ marks the lower-energy limit. We speculate then that $k A_{g}$ marks the upper Van Hove singularity; in this case $\delta E$ measures the width of the continuum band.

From the EA and TPA spectra and our SOS model, it is seen that two $A_{g}$ states above $1 B_{u}$ are strongly coupled to the $1 B_{u}$ exciton in luminescent polymers, namely, the $m A_{g}$ and the $k A_{g}$, with approximate energies 0.7 and $1.3 \mathrm{eV}$, respectively, above the $1 B_{u}$. This may explain the transient photoinduced absorption (PA) spectra recently measured in luminescent conducting polymers. ${ }^{94}$ Since the primary excitations in luminescent conducting polymers are $1 B_{u}$-type excitons, 
their strongest optical transitions should be to the $m A_{g}$ and $k A_{g}$ states, at $E_{b}$ and $E_{b}+\delta E$, respectively. Indeed, the picosecond transient PA spectra of luminescent polymers contain two strong PA bands at 0.8 and $1.4 \mathrm{eV}$, respectively, ${ }^{94}$ in good agreement with our prediction from the EA spectra.

Two important parameters characterizing the $1 B_{u}$ excitons can be calculated from the best fitting parameters of the EA spectra; they are also given in Tables III and IV. The first parameter is the Huang-Rhys parameter $S=1 / 2\left(\Delta q_{1}\right)^{2}$, which directly influences the shape of the optical absorption $1 A_{g} \rightarrow 1 B_{u}$ through phonon replica. In fact, $S$ determines the strength of the successive phonon replica in $\alpha(\omega)$, where

$$
\alpha(\omega)=A \operatorname{Im}\left[e^{-S} \mu_{01}^{2} \sum_{p=0}^{\infty} \frac{S^{p}}{p !} \frac{1}{\left(E_{1 B_{u}}+p \nu-\omega\right)}\right]
$$

We found $S<1$ for all polymers studied here, explaining the appearance of only three phonon replicas in many of their $\alpha(\omega)$ and PL spectra. ${ }^{72}$ The second parameter, which can be calculated from the fitting parameters, is the $1 B_{u}$ relaxation energy $E_{r}=S h \nu . E_{r}$ determines the apparent PL Stokes shift and figures in many theoretical models. We found $E_{r}$ to be of order $100 \mathrm{meV}$ for most polymers (Tables III and IV) in good agreement with the experiments. ${ }^{72,95} \mathrm{We}$ also found $E_{r}$ to be somewhat higher for nonluminescent polymers.

Other treatments of EA spectra through calculation of $\chi^{(3)}$ via SOS models ${ }^{30,79}$ are not as complete as ours here, since they either do not include phonon coupling ${ }^{30,79}$ or they use a simpler model based on the Huang-Rhys parameter alone. ${ }^{61}$ These models are not sufficient since the phonon coupling of the $1 B_{u} \rightarrow m A_{g}$ transition cannot be described in this way. If the configuration coordinate position of $m A_{g}$ is not equal to that of $1 B_{u}$ (which is the case in most polymers studied in this work) the amplitude of all phonon sidebands in the pathway $1 A_{g} \rightarrow 1 B_{u} \rightarrow m A_{g} \rightarrow 1 B_{u} \rightarrow 1 A_{g}$ for evaluating $\chi^{(3)}$ cannot be properly calculated by the Huang-Rhys approximation and may even have the wrong sign. In other treatments, the conjugation-length-related energy distribution is oversimplified to a plain Gaussian ${ }^{47}$ or not used at all. ${ }^{79}$ The latter case might lead to a misinterpretation of the EA spectral features. Both conjugation length distribution and application of vibrational levels show essential influence on the EA spectra. The analysis presented in this paper allows a more complete treatment of disordered polymer films with broad conjugation length distributions.

\section{EXCITON POLARIZABILITY CALCULATION}

The exciton polarizability $\Delta p$ can, in principle, be calculated from the EA spectrum using the first derivative spectrum of $\alpha(\omega)$ and $d \alpha / d E .{ }^{27,28,70}$ In our EA measurements the electric field was applied perpendicular to the direction of light propagation and the polymer chromophores were randomly oriented in the film. Averaging over all orientations of the polymers and assuming that $\Delta p$ and the transition dipole moments are parallel to the polymer backbone direction, it is found ${ }^{96}$ for the change $\Delta \alpha_{\mathrm{EA}}$ of the exciton absorption spectrum (the EA Stark shift of the exciton):

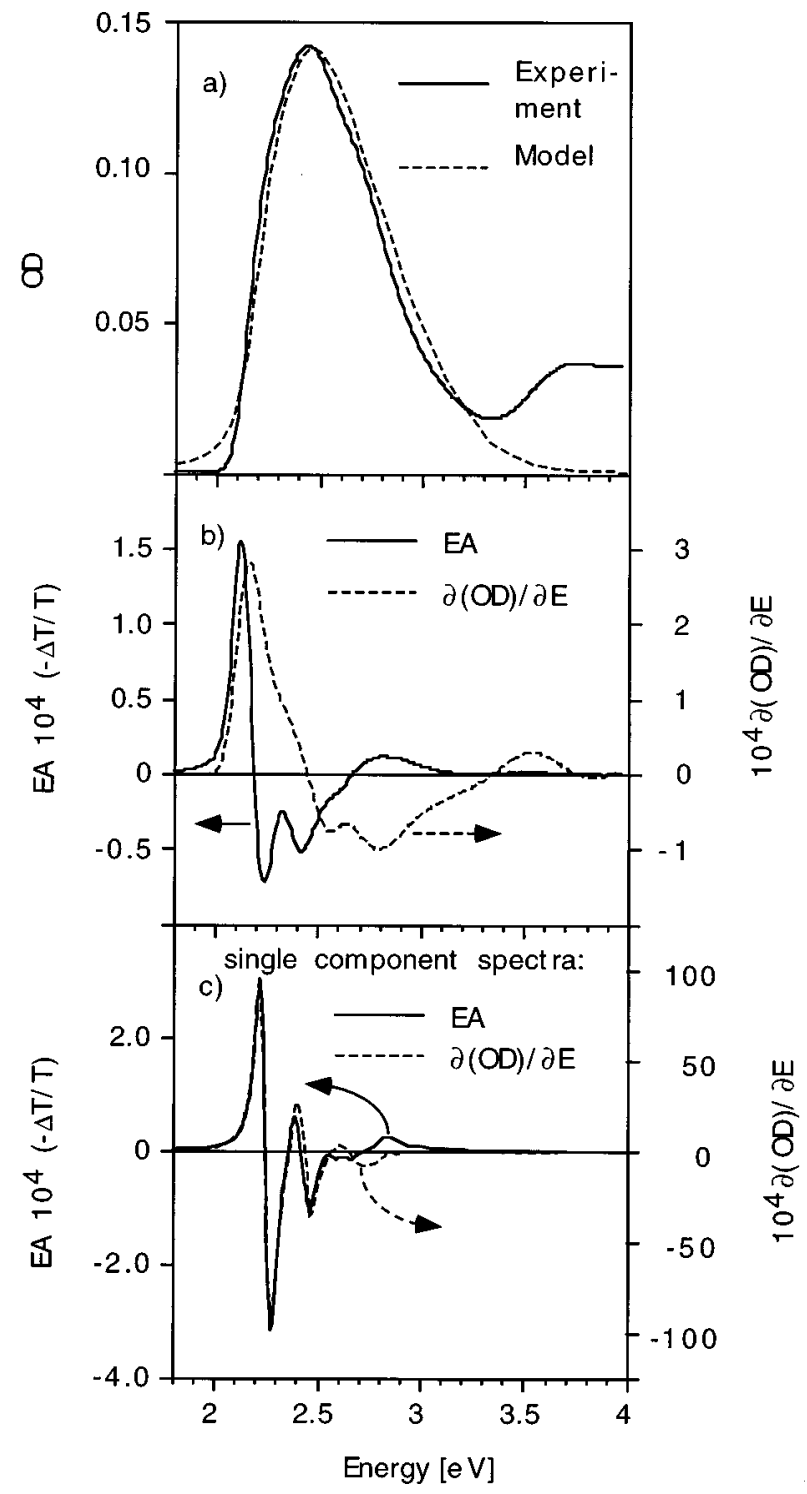

FIG. 10. The optical absorption (a), EA $\left(F=10^{5} \mathrm{~V} / \mathrm{cm}\right)(\mathrm{b})$ and one component EA (c) spectra of an MEH-PPV film at $80 \mathrm{~K}$ (full lines) compared with model calculations (dashed lines). The model in (a) uses the weight function $\widetilde{W}^{(2)}\left(E^{\prime}\right)$ to calculate the $\alpha(\omega)$ spectrum (see text); in (b) a comparison between EA (left axis) and $\partial \alpha / \partial \omega$ (right axis) spectra is made and in (c) a comparison of a one-component EA and $\partial \alpha / \partial \omega$ is made for $E\left(1 B_{u}\right)=2.2 \mathrm{eV}$.

$$
\Delta \alpha_{\mathrm{EA}}=\frac{1}{10} \Delta p F^{2} \frac{\partial \alpha}{\partial E}
$$

More sophisticated orientational averaging yields a similar result. ${ }^{61,70}$ Unfortunately, Eq. (25) cannot be directly used in most films. An example of the spectra $\alpha(\omega), d \alpha / d E$ and EA is given for MEH-PPV in Fig. 10. Clearly, the EA and $\partial \alpha / \partial E$ spectra do not match at high energies [Fig. 10(b)]. The reason for this discrepancy is the conjugation length distribution in the film and in particular, the dependence of $\Delta p$ on the conjugation length. From $\chi^{(3)}$ dependence on $N$ we know that $\Delta p$ is not constant but increases with the number of repeat units in the chain. Thus, even if Eq. (25) holds for the excitons of each conjugation length, since $\Delta p$ de- 


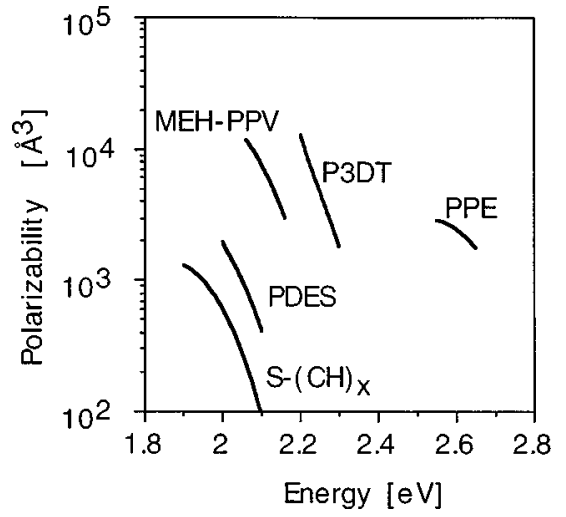

FIG. 11. The calculated electronic polarizability $\Delta p$ for the $1 B_{u}$ exciton in several luminescent (MEH-PPV, P3DT, and PPE) and nonluminescent $\left[s-(\mathrm{CH})_{x}\right.$ and PDES $] \pi$-conjugated polymers, as a function of $E\left(1 B_{u}\right) . \Delta p$ was estimated from the decomposition and comparison between the EA and $\partial \alpha / \partial \omega$ spectra (see text).

pends on $N$, the correlation between EA and $\partial \alpha / \partial E$ expressed in Eq. (25) for the entire polymer conjugation ensemble, is lost.

To overcome this difficulty, we may use two distribution functions representing the polymer conjugation length ensemble: $\widetilde{W}^{(2)}\left(E^{\prime}\right)$ describing the EA spectrum (previously discussed in Sec. IV) and $\widetilde{W}^{(1)}\left(E^{\prime}\right)$ describing $\alpha(\omega)$. For each conjugation length with $1 B_{u}$ energy $E^{\prime}$ we calculated the spectrum $\mathrm{EA}_{E^{\prime}}(\omega)$, via the SOS model (Sec. IV C) and the absorption spectra $\alpha_{E^{\prime}}(\omega)$ via Eq. (24), using the proper parameters from Tables I and II. The best $\widetilde{W}^{(1)}\left(E^{\prime}\right)$ was thus determined to fit the entire $\alpha(\omega)$ spectrum [see, for example, Fig. 10(a) for MEH-PPV]. We then calculated the spectrum of $(\partial \alpha / \partial E)_{E^{\prime}}$ for each $E^{\prime}$ and used Eq. (25) above to calculate $\Delta p\left(E^{\prime}\right)$ by comparing $\partial \alpha / \partial E$ to the EA spectrum from each $E^{\prime} .{ }^{32}$ An example for this procedure is given for MEHPPV in Fig. 10(c), where we indeed found that the calculated spectra $\mathrm{EA}_{E^{\prime}}(\omega)$ and $(\partial \alpha / \partial E)_{E^{\prime}}$ fit each other for the energy $E^{\prime}=2.2 \mathrm{eV}$ used in this example. In general, we found that $\mathrm{EA}_{E^{\prime}}$ and $(\partial \alpha / \partial E)_{E^{\prime}}$ spectra match each other for energies $E^{\prime}$ in an energy interval of $0.1 \mathrm{eV}$ from the minimum $E^{\prime}$ in the $\widetilde{W}\left(E^{\prime}\right)$ distributions.

The functions $\Delta p\left(E^{\prime}\right)$ thus obtained for three luminscent polymers (MEH-PPV, P3DT, and PPE) and two nonluminescent polymers $\left[s-(\mathrm{CH})_{x}\right.$ and PDES] are shown in Fig. 11. The function $\Delta p$ reaches its maximum value, $\Delta p_{\max }$, at the smallest $E^{\prime}$ for each polymer, which should be related with the longest respective conjugation in the conjugation length distribution. Away from $E^{\prime}$ minimum, $\Delta p\left(E^{\prime}\right)$ steeply decreases for all polymers. We note that $\Delta p_{\max }$ in luminescent polymers $\left(\simeq 10^{4} \AA\right)$ is about two orders of magnitude larger than $\Delta p_{\max }$ inferred from the EA spectra of polysilanes $\left[\Delta p_{\max } \simeq 180(\AA)^{3}\right],{ }^{96}$ and an order of magnitude larger than $\Delta p$ measured in PDA single crystal $\left[\Delta p \simeq 1500(\AA)^{3}\right] .^{32}$ This shows that $\pi$-electron delocalization in luminescent conducting polymers is surprisingly larger than both polysilanes and nonluminescent $\pi$-conjugated polymers, even in the form of single crystals.
$\Delta p_{\max }$ for the two polymers, MEH-PPV and P3DT, are especially interesting since other measurements of $\Delta p$ exist in the literature, using an entirely different experimental technique, namely, subnanosecond transient photoconductivity (TPC). In these measurements, ${ }^{97}$ an ultrafast TPC component was observed for the first $\sim 100 \mathrm{ps}$, which was interpreted as due to hot-carrier contributions to the photoconductivity. An alternative explanation was also given, where the ultrafast TPC was interpreted as due to a displacement current of the photogenerated excitons. ${ }^{98}$ In this case, it was possible to calculate the exciton polarizability using the TPC data from the photoexcitation density, assuming that the quantum efficiency of exciton generation is of order unity. Importantly, the estimated values of $\Delta p$ from TPC measurements using these assumptions are in excellent agreement with $\Delta p_{\max }$ extracted from the decomposition of the EA spectra in this work. $\Delta p$ was estimated from TPC (Ref. 98) to be $10^{4}(\AA)^{3}$ and $8 \times 10^{3} \AA^{3}$ for MEH-PPV and P3DT, respectively, while $\Delta p_{\max }$ extracted from EA spectra of these polymers is $1.2 \times 10^{4}(\AA)^{3}$ (Fig. 11). This agreement strongly indicates that the fast TPC component in conducting polymer films is indeed caused by the displacement current of photogenerated excitons, rather than by hot carriers. This may further justify the use of the exciton model ${ }^{68}$ over the band mode $l^{47}$ to describe the electronic states and photoexcitations in $\pi$-conjugated polymer thin films.

\section{CONCLUSIONS}

A SOS model was developed describing the measured EA spectra in a variety of luminescent and nonluminescent $\pi$ conjugated polymers. It consists of the third-order optical susceptibility $\operatorname{Im}\left[\chi^{(3)}(-\omega ; \omega, 0,0)\right]$, three essential states and their strongly coupled vibrations, and a conjugation length distribution of the excited-states energies. This SOS model allows us to fit equally well the experimental EA spectra of luminescent and nonluminescent conducting polymers. This approach is strengthened by the fact that the conjugationlength distribution can be used to simultaneously explain not only the rich, feature-laden EA spectrum, but also the relatively broad and featureless absorption spectrum. We found that $E\left(m A_{g}\right) / E\left(1 B_{u}\right) \cong 1.3$ for both luminescent and nonluminescent conducting polymers. The electron-phonon coupling (Huang-Rhys parameter $S$ ) was found to be smaller for luminescent polymers than for nonluminescent polymers. The $2 A_{g}$ state, which is responsible for the nonradiative decay pathway of excitons in nonluminescent polymers, does not play any important role in the EA spectra of the materials examined. We demonstrated that the understanding of disordered polymer thin films requires a conjugation length related energy and polarizability distribution for the excitons. The treatment presented here provides a rather complete analysis of the EA spectra in disordered polymer films.

\section{ACKNOWLEDGMENTS}

We acknowledge P. Lane for his help with the experimental setup and various discussions. This work was supported in part by the DOE, Grant No. FG-03-96 ER 454900. 
*Present address: ISM-CNR Via P. Gobetti 101, 40129 Bologna, Italy.

${ }^{\dagger}$ Also at 4pi Analysis, Inc., 3500 Westgate Drive, Suite 403, Durham, NC 27707-2534.

${ }^{1}$ M. Cardona, Solid State Physics Supplement 11: Modulation Spectroscopy (Academic Press, New York, 1969).

${ }^{2}$ W. V. Houston, Phys. Rev. 57, 184 (1940).

${ }^{3}$ L. V. Keldysh, Zh. Eksp. Teor. Fiz. 34, 1138 (1958) [Sov. Phys. JETP 7, 788 (1958)].

${ }^{4}$ G. H. Wannier, Rev. Mod. Phys. 34, 645 (1962).

${ }^{5}$ K. Tharmalingam, Phys. Rev. 130, 2204 (1963).

${ }^{6}$ D. Redfield, Phys. Rev. 130, 914 (1963).

${ }^{7}$ D. Redfield, Phys. Rev. 140, A2056 (1965).

${ }^{8}$ J. Callaway, Phys. Rev. 130, 549 (1963).

${ }^{9}$ J. Callaway, Phys. Rev. 134, A998 (1964).

${ }^{10}$ R. Enderlein, Phys. Status Solidi 20, 295 (1967).

${ }^{11}$ R. Enderlein and R. Keiper, Phys. Status Solidi 23, 127 (1967).

${ }^{12}$ R. Enderlein and R. Keiper, Phys. Status Solidi 19, 673 (1967).

${ }^{13}$ D. F. Blossey, Phys. Rev. B 2, 3976 (1970).

${ }^{14}$ D. F. Blossey, Phys. Rev. B 3, 1382 (1971).

${ }^{15}$ Y. Hamakawa, F. A. Germano, and P. Handler, Phys. Rev. 167, 703 (1968).

${ }^{16}$ A. Frova and P. Handler, Phys. Rev. 137, A1857 (1965).

${ }^{17}$ A. Frova et al., Phys. Rev. 145, 575 (1966).

${ }^{18}$ P. Handler, Phys. Rev. 137, A1862 (1965).

${ }^{19}$ D. E. Aspnes, Phys. Rev. 147, 554 (1966).

${ }^{20}$ D. E. Aspnes, Phys. Rev. 153, 97 (1967).

${ }^{21}$ D. E. Aspnes, P. Handler, and D. F. Blossey, Phys. Rev. 166, 921 (1968)

${ }^{22}$ D. E. Aspnes and J. E. Rowe, Phys. Rev. B 5, 4022 (1972).

${ }^{23}$ D. E. Aspnes, Surf. Sci. 37, 418 (1973).

${ }^{24}$ D. E. Aspnes and J. E. Rowe, Solid State Commun. 8, 1145 (1970).

${ }^{25}$ B. O. Seraphin, Phys. Rev. 140, A1716 (1965).

${ }^{26}$ B. O. Seraphin and N. Bottka, Phys. Rev. 145, 628 (1966).

${ }^{27}$ L. Sebastian and G. Weiser, Chem. Phys. Lett. 64, 396 (1979).

${ }^{28}$ L. Sebastian and G. Weiser, Chem. Phys. 62, 447 (1981).

${ }^{29}$ L. Sebastian and G. Weiser, Phys. Rev. Lett. 46, 1156 (1981).

${ }^{30}$ D. Guo, S. Mazumdar, S. N. Dixit, F. Kajzar, F. Jarka, Y.

Kawabe, and N. Peyghambarian, Phys. Rev. B 48, 1433 (1993).

${ }^{31}$ D. Guo et al., Mol. Cryst. Liq. Cryst. 256, 105 (1994).

${ }^{32}$ G. Weiser, Phys. Rev. B 45, 14076 (1992).

${ }^{33}$ Á. Horváth, H. Bässler, and G. Weiser, Phys. Status Solidi B 173, 755 (1992).

${ }^{34}$ Á. Horváth, G. Weiser, G. L. Baker, and S. Etemad, Phys. Rev. B 51, 2751 (1995).

${ }^{35}$ Á. Horváth, G. Weiser, C. Lapersonne-Meyer, M. Schott, and S. Spagnoli, Phys. Rev. B 53, 13507 (1996).

${ }^{36}$ J. Orenstein, G. L. Baker, and Z. V. Vardeny, J. Phys. (Paris), Colloq. 44, C3-407 (1983).

${ }^{37}$ S. D. Phillips, R. Worland, G. Yu, T. Hagler, R. Freedman, V. Yoon, J. Chiang, W. C. Walker, and A. J. Heeger, Phys. Rev. B 40, 975 (1989).

${ }^{38}$ T. W. Hagler and A. J. Heeger, Phys. Rev. B 49, 7313 (1994).

${ }^{39}$ T. Nishioka, T. Yamada, S. Shibahara, K. Ishikawa, H. Takezoe, and A. Fukuda, Jpn. J. Appl. Phys., Part 1 36, 1099 (1997).

${ }^{40}$ F. Rohlfing, D. D. C. Bradley, A. Eberhardt, K. Muller, J. Cornil, D. Beljonne, and J. L. Bredas, Synth. Met. 76, 35 (1996).

${ }^{41}$ D. Beljonne, J. Cornil, Z. Shuai, J. L. Bredas, F. Rohlfing, D. D. C. Bradley, W. E. Torruelas, V. Ricci, and G. I. Stegeman, Phys. Rev. B 55, 1505 (1997).
${ }^{42}$ O. M. Gelsen, D. D. C. Bradley, H. Murata, N. Takada, T. Tsutsui, and S. Saito, J. Appl. Phys. 71, 1064 (1992).

${ }^{43}$ J. M. Leng, S. Jeglinski, X. Wei, R. E. Benner, Z. V. Vardeny, D. Guo, and S. Mazumdar, Phys. Rev. Lett. 72, 156 (1994).

${ }^{44}$ Y. Tokura, T. Koda, A. Itsubo, M. Miyabayashi, K. Okuhara, and A. Uedov, J. Chem. Phys. 85, 99 (1992).

${ }^{45}$ S. Jeglinski and Z. V. Vardeny, Synth. Met. 50, 509 (1992).

${ }^{46}$ S. J. Martin, O. M. Gelsen, D. D. C. Bradley, and C. Botta, Mol. Cryst. Liq. Cryst. 256, 591 (1994).

${ }^{47}$ T. W. Hagler, K. Pakbaz, and A. J. Heeger, Phys. Rev. B 49, 10968 (1994).

${ }^{48}$ L. M. Blinov, S. P. Palto, G. Ruani, C. Taliani, A. A. Tevesov, S. G. Yudin, and R. Zamboni, Chem. Phys. Lett. 232, 401 (1995).

${ }^{49}$ P. A. Lane, Z. V. Vardeny, M. Liess, J. Partee, J. Shinar, E. Ibrahim, and A. Frank, J. Phys. Chem. (to be published).

${ }^{50}$ R. Louden, Am. J. Phys. 27, 649 (1959).

${ }^{51}$ M. Shinada and S. Sugano, J. Phys. Soc. Jpn. 21, 1936 (1966).

${ }^{52}$ S. Abe, J. Phys. Soc. Jpn. 58, 62 (1989).

${ }^{53}$ T. Ogawa and T. Takagahara, Phys. Rev. B 43, 8138 (1991).

${ }^{54}$ T. Ogawa and T. Takagahara, Phys. Rev. B 43, 14325 (1991).

${ }^{55} \mathrm{H}$. Haug and S. W. Koch, Quantum Theory of the Optical and Electronic Properties of Semiconductors, 2nd ed. (World Scientific, Singapore, 1993).

${ }^{56}$ B. E. Kohler, in Conjugated Polymers, The Novel Science and Technology of Conducting and Nonlinear Optically Active Materials, edited by J. L. Brédas and R. Silbey (Kluwer Press, Dordrecht, 1991).

${ }^{57}$ D. Baeriswyl, D. K. Campbell, and S. Mazumdar, in Conjugated Conducting Polymers, edited by H. G. Kiess (Springer-Verlag, New York, 1992).

${ }^{58}$ D. Guo, S. Mazumdar et al., Phys. Rev. B 48, 1433 (1993).

${ }^{59}$ S. Abe, J. Yu, and W. P. Su, Phys. Rev. B 45, 8264 (1992).

${ }^{60} \mathrm{~S}$. Abe, in Relaxation in Polymers, edited by T. Kobayshi (World Scientific, Singapore, 1993).

${ }^{61}$ S. A. Jeglinski, Ph.D. thesis, University of Utah, 1996.

${ }^{62}$ Z. G. Soos, D. Mukhopadhyay, and M. H. Hennessy, Chem. Phys. 210, 249 (1996).

${ }^{63}$ B. J. Orr and J. F. Ward, Mol. Phys. 20, 53 (1971).

${ }^{64}$ A. Willetts, J. E. Rice, D. M. Burland, and D. P. Shelton, J. Chem. Phys. 97, 7590 (1992).

${ }^{65}$ Z. G. Soos, D. S. Galvao, and S. Etemad, Adv. Mater. 6, 280 (1994).

${ }^{66}$ J. Cornil et al., Chem. Phys. Lett. 233, 82 (1994).

${ }^{67}$ M. J. Rice and Y. N. Gartstein, Phys. Rev. Lett. 73, 2504 (1994).

${ }^{68}$ M. Chandross, S. Mazumdar, S. Jeglinski, X. Wei, Z. V. Vardeny, E. W. Kwock, and T. M. Miller, Phys. Rev. B 50, 14702 (1994).

${ }^{69}$ Y. N. Gartstein, M. J. Rice, and E. M. Conwell, Phys. Rev. B 51, 5546 (1995).

${ }^{70}$ S. Jeglinski, Z. V. Vardeny, Y. Ding, and T. Barton, Mol. Cryst. Liq. Cryst. 256, 87 (1994).

${ }^{71}$ S. Mazumdar and M. Chandross, in Optical and Photonic Applications of Electroactive and Conducting Polymers, edited by S. C. Yang and P. Chandrasekhar, SPIE Proc. No. 2528 (The International Society of Optical Engineering, San Diego, California, 1995), p. 62.

${ }^{72}$ K. Pickler, D. A. Halliday, D. D. C. Bradley, P. L. Burn, R. H. Friend, and A. B. Holmes, J. Phys.: Condens. Matter 5, 7155 (1993).

${ }^{73}$ M. Chandross, S. Mazumdar, M. Liess, P. A. Lane, Z. V. Vardeny, M. Hamaguchi, and K. Yoshino, Synth. Met. 84, 603 
(1997); Phys. Rev. B 55, 1486 (1997).

${ }^{74}$ Y. Shimoi and S. Abe, Synth. Met. 78, 219 (1996).

${ }^{75}$ M. Ozaki, M. Liess, R. E. Benner, Z. V. Vardeny, K. Yoshino, and R. Sugimoto, Synth. Met. 84, 511 (1997).

${ }^{76}$ J. R. Heflin, K. Y. Wong, O. Zamoni-Khamiri, and A. F. Garitos, Phys. Rev. B 38, 1573 (1988).

${ }^{77}$ P. C. M. McWilliams, G. W. Hayden, and Z. G. Soos, Phys. Rev. B 43, 9777 (1991).

${ }^{78}$ S. N. Dixit, D. Guo, and S. Mazumdar, Phys. Rev. B 43, 6781 (1991); J. Chem. Phys. 100, 1665 (1994).

${ }^{79}$ D. Mukhopadhay and Z. G. Soos, J. Chem. Phys. 104, 1600 (1996).

${ }^{80}$ C. Poga, T. M. Brown, M. G. Kuzyk, and C. W. Dirk, J. Opt. Soc. Am. B 12, 531 (1995).

${ }^{81}$ J. Kurti and H. Kuzmani, Phys. Rev. B 44, 597 (1991), and references therein.

${ }^{82}$ E. Ehrenfreund, Z. V. Vardeny, O. Brafman, and B. Horovitz, Phys. Rev. B 36, 1535 (1987).

${ }^{83}$ G. P. Brivio and E. Mulazi, Chem. Phys. Lett. 95, 555 (1983).

${ }^{84}$ J. P. Hermann and J. Ducuing, J. Appl. Phys. 45, 5100 (1974).

${ }^{85}$ Z. Shuai and J. L. Brédas, Phys. Rev. B 44, 5962 (1991).

${ }^{86}$ H. Thienpont et al., Phys. Rev. Lett. 65, 2141 (1990).

${ }^{87}$ L. P. Cheng et al., J. Phys. Chem. 95, 10643 (1991).

${ }^{88}$ Preliminary SOS model calculation of several nonluminescent polymers was published in M. Liess, S. Jeglinski, P. A. Lane, and Z. V. Vardeny, Synth. Met. 84, 891 (1997).

${ }^{89}$ J. B. Van Beek, F. Kajzar, and A. C. Albrecht, J. Chem. Phys. 95, 6400 (1991).

${ }^{90}$ R. K. Meyer, R. E. Benner, M. Ozaki, M. Liess, Z. V. Vardeny, K. Yoshino, Y. Ding, and T. Barton, Synth. Met. 84, 549 (1997).

${ }^{91}$ Á. Horváth, G. Weiser, C. Lapersonne Meyer, M. Schott, and S. Spagnoli, Synth. Met. 84, 553 (1997).

${ }^{92}$ P. Gomes da Costa and E. Conwell, Phys. Rev. B 48, 1993 (1993).

${ }^{93}$ A. J. Heeger, S. Kivelson, J. R. Schrieffer, and W. P. Su, Rev. Mod. Phys. 60, 781 (1988).

${ }^{94}$ S. V. Frolov, W. Gellermann, Z. V. Vardeny, M. Ozaki, and K. Yoshino, Synth. Met. 84, 493 (1997); Phys. Rev. Lett. 78, 4285 (1997).

${ }^{95}$ C. M. Heller, I. H. Campbell, B. K. Laurich, D. L. Smith, D. D. C. Bradley, P. L. Burn, J. P. Ferraris, and K. Mullen, Phys. Rev. B 54, 5516 (1996).

${ }^{96}$ R. G. Kepler and Z. G. Soos, Phys. Rev. B 43, 12530 (1991).

${ }^{97}$ C. H. Lee, G. Yu, D. Moses, and A. J. Heeger, Phys. Rev. B 49, 2396 (1994).

${ }^{98}$ D. Moses, D. Comoretto, C. H. Lee, and A. J. Heeger, Synth. Met. 84, 559 (1997). 